\title{
Prevalence of Non-Alcoholic Fatty Liver Disease and its Association with Diabetic Nephropathy in Type 2 Diabetes Mellitus Patients
}

\author{
Laltesh Kumar ${ }^{1}$, Alok Kumar ${ }^{2}$ \\ ${ }^{1}$ Assistant Professor, Department of Medicine, Varun Arjun Medical College, Banthra, Shahjahanpur, UP, ${ }^{2}$ Assistant Professor, \\ Department of Medicine, Varun Arjun Medical College, Banthra, Shahjahanpur, UP, India
}

Corresponding author: Laltesh Kumar, Assistant Professor, Department of Medicine, Varun Arjun Medical College, Banthra, Shahjahanpur, UP, India

DOI: http://dx.doi.org/10.21276/ijcmsr.2020.5.1.53

How to cite this article: Laltesh Kumar, Alok Kumar. Prevalence of non-alcoholic fatty liver disease and its association with diabetic nephropathy in type 2 diabetes mellitus patients. International Journal of Contemporary Medicine Surgery and Radiology. 2020;5(1):A243-A246.

\section{A B S T R A C T}

Introduction: Diabetes mellitus (DM) is a group of metabolic diseases characterized by hyperglycemia resulting from defect in insulin secretion, insulin action or both. The association between Non Alcoholic Fatty Liver Disease (NAFLD) and type 2 diabetes mellitus (T2DM) has been well established. Hence the present study was carried for assessing the prevalence of non-alcoholic fatty liver disease and its association with diabetic nephropathy in type 2 diabetes mellitus patients.

Material and methods: A total of 150 type 2 diabetic patients were enrolled in the present study. Complete demographic and clinical details of all the patients were obtained. Blood samples were obtained from all the patients and routine investigations were carried out. Hepatic ultrasonography was performed in all patients after 12 hours fasting. All the results were recorded in Microsoft excel sheet and were analysed by SPSS software. Chi- square test was used for evaluation of level of significance.

Results: NAFLD was found to be present in 109 patients. Therefore; the overall prevalence of NAFLD was found to be 72.67 percent of the patients. The overall prevalence of diabetic nephropathy was found to be 32.66 percent among diabetic patients. Out of 49 patients with diabetic nephropathy, NAFLD was found to be present in 35 patients while it was absent in 14 patients. While assessing the association of NAFLD with diabetic nephropathy in type 2 diabetic patients, non-significant results were obtained.

Conclusion: NAFLD was not found to be a significant risk factor for development of diabetic nephropathy in type 2 diabetic patients.

Keywords: Non-alcoholic Fatty Liver, Diabetic Nephropathy

\section{INTRODUCTION}

Diabetes mellitus (DM) is a group of metabolic diseases characterized by hyperglycemia resulting from defect in insulin secretion, insulin action or both. It is an important cause of morbidity and mortality worldwide. ${ }^{1}$ The reninangiotensin system plays a predominant role in the evolution of renal disease, not only by inducing changes in arterial pressure (BP) and glomerular hemodynamics, but also by increasing oxidative stress and promoting expression of proinflammatory genes and pro-fibrotic factors in mesangial cells and vascular smooth muscle, additionally, angiotensin II promotes mesangial cell and vascular smooth muscle proliferation, thus contributing to the enlargement of the arteriolar wall. Activated inflammatory cells, especially $\mathrm{T}$ cells, synthesize angiotensin, thereby reinforcing intrarenal angiotensin production. ${ }^{2-4}$

The association between NAFLD and type 2 diabetes mellitus (T2DM) has been well established, which could be explained by the insulin-resistance and compensatory hyperinsulinemia progressing to defective lipid metabolism and hepatic triglyceride (TG) accumulation in NAFLD or to $\beta$-cell dysfunction in T2DM. Compared with nondiabetic subjects, patients with T2DM appear to have an increased risk of developing NAFLD and certainly have a heightened risk of developing advanced liver diseases, such as fibrosis, cirrhosis, and hepatocellular carcinoma. ${ }^{5-7}$ Hence the present study was carried for assessing the prevalence of non-alcoholic fatty liver disease and its association with diabetic nephropathy in type 2 diabetes mellitus patients.

\section{MATERIAL AND METHODS}

The present study was conducted in the department of medicine from Oct 2019 to Dec 2019 and it included assessment of the prevalence of non-alcoholic fatty liver disease and its association with diabetic nephropathy in type 2 diabetes mellitus patients. Ethical approval was obtained 
from institutional ethical committee and written consent was obtained from all the patients after explaining in detail the entire research protocol. A total of 150 type 2 diabetic patients were enrolled in the present study. Exclusion criteria for the present study included:

- Patients who didn't gave the informed consent,

- Patients with positive history of any known drug allergy,

- $\quad$ Patients with presence of any malignant neoplasm Complete demographic and clinical details of all the patients were obtained. Blood samples were obtained from all the patients and routine investigations were carried out. Hepatic ultrasonography was performed in all patients after 12 hours fasting, and fatty liver based on standard criteria was diagnosed. Diagnosis of diabetic nephropathy was done by a positive persistent albuminuria for a minimum of three consecutive readings within the study. The prevalence of NAFLD and diabetic nephropathy was assessed among diabetic patients. All the results were recorded in Microsoft excel sheet and were analysed by SPSS software. Chi- square test was used for evaluation of level of significance. P- value of less than 0.05 was taken as significant.

\section{RESULTS}

In the present study, a total of 150 type 2 diabetic patients were enrolled. Mean age of the diabetic patients was found to be 41.5 years. 56 patients belonged to the age group of 30 to 45 years. 89 patients were males while the remaining were females. 96 patients had rural residence while the remaining
54 patients had urban residence (table-1). NAFLD was found to be present in 109 patients. Therefore; the overall prevalence of NAFLD was found to be 72.67 percent of the patients (table-2).

In the present study, the overall prevalence of diabetic nephropathy was found to be 32.66 percent among diabetic patients (table-3). Out of 49 patients with diabetic nephropathy, NAFLD was found to be present in 35 patients while it was absent in 14 patients. While assessing the association of NAFLD with diabetic nephropathy in type 2 diabetic patients, non-significant results were obtained (table-4, graph-1).

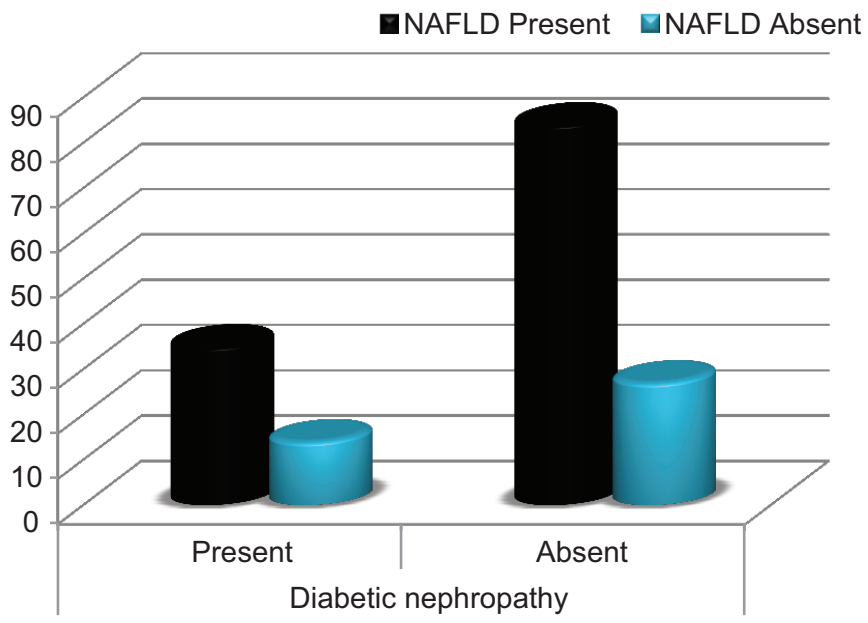

Graph-1: Correlation of NAFLD with diabetic nephropathy in type 2 diabetic patients

\begin{tabular}{|l|l|c|}
\hline \multicolumn{2}{|l|}{ Parameter } & Number \\
\hline \multicolumn{2}{|l|}{ Number of patients } & 150 \\
\hline \multirow{3}{*}{ Age group (years) } & Less than 30 & 42 \\
\cline { 2 - 3 } & 30 to 45 & 56 \\
\cline { 2 - 3 } & More than 45 & 52 \\
\hline \multirow{2}{*}{ Gender } & Males & 69 \\
\cline { 2 - 3 } & Females & 91 \\
\hline \multirow{2}{*}{ Residence } & Rural & 96 \\
\cline { 2 - 3 } & Urban & 54 \\
\hline
\end{tabular}

\begin{tabular}{|l|c|c|}
\hline Parameter & Number & Percentage \\
\hline Prevalence of NAFLD & 109 & 72.67 \\
\hline \multicolumn{2}{|c|}{ Table-2: Prevalence of non-alcoholic fatty liver disease in diabetic patients } \\
\hline
\end{tabular}

\begin{tabular}{|l|c|c|}
\hline Parameter & Number & Percentage \\
\hline Prevalence of diabetic nephropathy & 49 & 32.66 \\
\hline \multicolumn{2}{|c|}{ Table-3: Prevalence of diabetic nephropathy in diabetic patients } \\
\hline
\end{tabular}

\begin{tabular}{|c|c|c|c|c|c|c|}
\hline \multicolumn{2}{|c|}{ Parameter } & \multicolumn{2}{|c|}{ Diabetic nephropathy } & \multirow{3}{*}{\begin{tabular}{|c|} 
Total \\
109
\end{tabular}} & \multirow{3}{*}{$\begin{array}{c}\begin{array}{c}\text { Chi- square } \\
\text { value }\end{array} \\
0.3222\end{array}$} & \multirow{3}{*}{$\begin{array}{c}\text { p-value } \\
0.5704\end{array}$} \\
\hline & & \multirow{2}{*}{$\begin{array}{c}\text { Present } \\
35\end{array}$} & \multirow{2}{*}{$\begin{array}{c}\text { Absent } \\
84\end{array}$} & & & \\
\hline NAFLD & Present & & & & & \\
\hline & Absent & 14 & 27 & 41 & & \\
\hline \multicolumn{2}{|l|}{ Total } & 49 & 101 & 150 & & \\
\hline
\end{tabular}




\section{DISCUSSION}

NAFLD, defined as the presence of hepatic steatosis in the absence of secondary causes, is emerging as a public health issue worldwide, with a global pooled prevalence, by imaging, of $25.24 \%$ among general population. NAFLD includes a spectrum of diseases ranging from simple steatosis to nonalcoholic steatohepatitis (NASH), and to advanced fibrosis and cirrhosis and hepatocellular carcinoma, causing considerable liver-related morbidity and mortality. ${ }^{7-9}$ Hence: the present study was carried for assessing the prevalence of non-alcoholic fatty liver disease and its association with diabetic nephropathy in type 2 diabetes mellitus patients.

In the present study, a total of 150 type 2 diabetic patients were enrolled. Mean age of the diabetic patients was found to be 41.5 years. NAFLD was found to be present in 109 patients. Therefore; the overall prevalence of NAFLD was found to be 72.67 percent of the patients. Heidari $Z$ et al investigated the association between Non Alcoholic Fatty Liver Disease (NAFLD) and diabetic nephropathy in these patients. This cross-sectional study was conducted on 255 patients with T2DM, with minimum age being 30 years. Hepatic ultrasonography using a $3.5 \mathrm{MHz}$ probe was performed in all subjects. Fatty liver based on standard criteria was diagnosed with liver brightness, contrast between the echogenicity of the liver, kidneys and the blood vessels fading rate. Screening for microalbuminuria was performed by the preferred method, measurement of the Urine Albumin-ToCreatinine (UACR) ratio in a random spot collection. 255 patients with T2DM were enrolled of which 173 (68\%) were females and $82(32 \%)$ were males. Of these 221 subjects (86.66\%) had NAFLD. Diabetic nephropathy was observed among 33\% of individuals, microalbuminuria among 32\% and macroalbuminuria in 10\% of all individuals. Duration of diabetes, Body Mass Index (BMI), hypertriglyceridemia, and $\mathrm{HbA1c}$ were significantly associated with incidence of NAFLD. Also, duration of diabetes and $\mathrm{HbA1c}$ were significantly associated with diabetic nephropathy in patients with T2DM. NAFLD in patients with T2DM is extremely common. ${ }^{10}$

In the present study, the overall prevalence of diabetic nephropathy was found to be 32.66 percent among diabetic patients. Out of 49 patients with diabetic nephropathy, NAFLD was found to be present in 35 patients while it was absent in 14 patients. While assessing the association of NAFLD with diabetic nephropathy in type 2 diabetic patients, non-significant results were obtained. Dai W et al estimated the pooled prevalence of NAFLD in T2DM patients. Electronic databases of PubMed, Web of Science, Embase, Chinese National Knowledge Infrastructure, and Wanfang were searched using $\mathrm{MeSH}$ terms to identify relevant studies. Eligibility assessment and data extraction were conducted independently by 2 investigators and a metaanalysis was performed to synthesize the data. Heterogeneity was evaluated using the Cochran $Q$ test and quantified using the I2 statistic. Publication bias was assessed using both the Begg and Egger tests. Subgroup analyses were performed to identify the possible sources of heterogeneity. Twenty-four studies involving 35,599 T2DM patients were included in this meta-analysis, of which 20,264 were identified with NAFLD. A high degree of heterogeneity (I2=99.0\%, $\mathrm{P}<.001$ ) was observed among the eligible studies, with the reported prevalence ranging from $29.6 \%$ to $87.1 \%$. The pooled prevalence of NAFLD in T2DM patients, by a random-effects model, was $59.67 \%$. Sensitivity was low and both the Begg test and Egger test showed low possibility of publication bias. Subgroup analyses indicated that the prevalence of NAFLD in T2DM patients differed by gender, obesity, hypertension, dyslipidemia, coronary heart disease, and chronic kidney disease. The high pooled prevalence of NAFLD in T2DM patients found in this study significantly underscores the need for early assessment of NAFLD and the importance of strengthening the management of NAFLD in T2DM patients. ${ }^{11}$

The clinical associations of NAFLD with the element of metabolic syndrome, including obesity, hypertension, and dyslipidemia have been well established. ${ }^{12}$ For example, Yi et al found that body mass index and dyslipidemia were independent risk factors for NAFLD in T2DM, ${ }^{13}$ and Leite et al found that the occurrence of NAFLD in T2DM was associated with obesity and hypertriglyceridemia. ${ }^{14}$ Additionally, Ding et al found that T2DM patients with NAFLD had significantly higher levels of systolic blood pressure and diastolic blood pressure than T2DM patients without NAFLD. ${ }^{15}$ Based on these findings, it was deduced that NAFLD may be a hepatic manifestation of metabolic syndrome. Consistently, the prevalence of NAFLD in T2DM patients differed significantly with differences in sample characteristics, including obesity, hypertension, and dyslipidemia. Insulin resistance, which is highly shared in the element of metabolic syndrome, could, to a large extent, account for the associations of obesity, dyslipidemia, and hypertension with NAFLD in T2DM. ${ }^{16}$

\section{CONCLUSION}

From the above results, the authors concluded that NAFLD was not found to be a significant risk factor for development of diabetic nephropathy in type 2 diabetic patients. However; further studies are recommended.

\section{REFERENCES}

1. Lutale JJ, Thordarson H, Abbas ZG, Vetvik K. Microalbuminuria among type 1 and type 2 diabetic patients of African origin in Dar Es Salaam, Tanzania. BMC Nephrol. 2007;8(1):2.

2. Ritz E. Nephropathy in type 2 diabetes. J Intern Med. 1999;245(3):111-26.

3. Molitch ME, DeFronzo RA, Franz MJ, Keane WF, Mogensen CE, Parving $\mathrm{HH}$, et al. Nephropathy in diabetes. Diabetes Care. 2004;27(Suppl 1):S79-83.

4. Battisti WP, Palmisano J, Keane WE. Dyslipidemia in patients with type 2 diabetes. Relationship between lipids, kidney disease and cardiovascular disease. Clin Chem Lab Med. 2003;41(5):1174-81.

5. Younossi ZM, Gramlich T, Matteoni CA, et al. Nonalcoholic fatty liver disease in patients with type 2 diabetes. Clin Gastroenterol Hepatol 2004;2(1):262-5.

6. Targher G, Bertolini L, Rodella S, et al. Nonalcoholic fatty liver disease is independently associated with an increased incidence of cardiovascular events in type 2 
diabetic patients. Diabetes Care 2007;30(2):2119-21.

7. Parving HH, Gall MA, Skøtt P, Jørgensen HE, Løkkegaard H, Jørgensen F, et al. Prevalence and causes of albuminuria in non-insulin-dependent diabetic patients. Kidney Int. 1992;41(4):758-62.

8. Leite NC, Villela-Nogueira CA, Pannain VL, et al. Histopathological stages of nonalcoholic fatty liver disease in type 2 diabetes: prevalences and correlated factors. Liver Int 2011;31(6):700-6.

9. Targher G, Bertolini L, Rodella S, et al. Non-alcoholic fatty liver disease is independently associated with an increased prevalence of chronic kidney disease and proliferative/laser-treated retinopathy in type 2 diabetic patients. Diabetologia 2008;51(3):444-50

10. Heidari Z, Gharebaghi A. Prevalence of Non Alcoholic Fatty Liver Disease and its Association with Diabetic Nephropathy in Patients with Type 2 Diabetes Mellitus. J Clin Diagn Res. 2017;11(5):OC04-OC07.

11. Dai W, Ye L, Liu A, et al. Prevalence of nonalcoholic fatty liver disease in patients with type 2 diabetes mellitus: A meta-analysis. Medicine (Baltimore). 2017;96(39):e8179.

12. Kalra S, Vithalani M, Gulati G, et al. Study of prevalence of nonalcoholic fatty liver disease (NAFLD) in type 2 diabetes patients in India (SPRINT). J Assoc Physicians India 2013;61(5):448-53

13. Yi M, Chen RP, Yang R, et al. Increased prevalence and risk of non-alcoholic fatty liver disease in overweight and obese patients with Type 2 diabetes in South China. Diabetic Med 2017;34(2):505-13.

14. Leite NC, Salles GF, Araujo ALE, et al. Prevalence and associated factors of non-alcoholic fatty liver disease in patients with type- 2 diabetes mellitus. Liver Int 2009;29(6):113-9.

15. Ding X, Xu Y, Wang Y, et al. Nonalcoholic fatty liver disease and associated metabolic risks of hypertension in type 2 diabetes: a cross-sectional community-based study. Int J Endocrinol 2017;2017:526256.

16. Wu JC, Li XH, Peng YD, et al. Non-alcoholic fatty liver disease is associated with chronic kidney disease and diabetic retinopathy in type 2 diabetic patients. Chin J Pract Intern Med 2010;30(5):41-3.

Source of Support: Nil; Conflict of Interest: None

Submitted: 11-01-2020; Accepted: 16-02-2020; Published online: 20-03-2020 